\title{
ENCHENTES E VAZANTES DO RIO NEGRO MEDIDAS NO PORTO DE MANAUS, AMAZONAS, BRASIL
}

\author{
José Alberto NUNES DE MELLO', Wanilze Gonçalves BARROS ${ }^{2}$
}

\begin{abstract}
RESUMO - As cotas do rio Negro vem sendo medidas, diariamente, desde setembro de 1902. O presente trabalho baseou-se em dados colhidos entre os anos de 1903 a 1999. As cinco maiores enchentes aconteceram em: $1953(29,69 \mathrm{~m}), 1976(29,61 \mathrm{~m}), 1989(29,42 \mathrm{~m}), 1922(29,35 \mathrm{~m}) \mathrm{e}$ $1909(29,17 \mathrm{~m})$. As cinco maiores vazantes aconteceram em: $1963(13,65 \mathrm{~m}), 1906(14,20 \mathrm{~m})$, $1997(14,34 \mathrm{~m}), 1916(14,42 \mathrm{~m})$ e $1926(14,54 \mathrm{~m})$. Os cinco menores valores de cotas máximas foram em: $1926(21,77 \mathrm{~m}), 1912(24,84 \mathrm{~m}), 1992(25,42 \mathrm{~m}), 1964(25,91 \mathrm{~m})$ e $1980(26,00 \mathrm{~m})$ e os cinco de cotas mínimas foram em: 1926 (21,77m), $1912(24,84 \mathrm{~m}), 1992(25,42 \mathrm{~m}), 1964(25,91 \mathrm{~m})$ e $1980(26,00 \mathrm{~m})$. As cinco maiores diferenças alcançadas em um mesmo ano entre as cotas máximas e mínimas aconteceram em : 1997 (14,62m), $1909(14,13 \mathrm{~m}), 1953(12,62 \mathrm{~m}), 1952$ $(12,44 \mathrm{~m})$ e $1916(12,21 \mathrm{~m})$ e as menores aconteceram em: $1912(05,45 \mathrm{~m}), 1968(06,10 \mathrm{~m}), 1985$ $(06,53 \mathrm{~m}), 1974,(06,62 \mathrm{~m})$ e $1986(06,74 \mathrm{~m})$.
\end{abstract}

Palavras-chave: Enchentes, Vazantes, Rio-Negro

\section{Rio Negro Water Level Fluctuations at the Manaus Harbor, Amazonas, Brazil}

ABSTRACT - The quotes of the Negro River are measured every day since September 1902 until today. This work is based on the years 1903 until 1999. The five highest flooding happened in: $1953(29,69 \mathrm{~m}), 1976(29,61 \mathrm{~m}), 1989(29,42 \mathrm{~m}), 1922(29,35 \mathrm{~m})$ and $1909(29,17 \mathrm{~m})$. The five lowest floding happened in: $1963(13,65 \mathrm{~m}), 1906(14,20 \mathrm{~m}), 1997(14,34 \mathrm{~m}), 1916$ $(14,42 \mathrm{~m})$ and $1926(14,54 \mathrm{~m})$. The five smaller values of maximum quotas were em:1926 $(21,77 \mathrm{~m}), 1912(24,84 \mathrm{~m}), 1992(25,42 \mathrm{~m}), 1964(25,91 \mathrm{~m})$ and $1980(26,00 \mathrm{~m})$ and the five of minimum quotas were in: $1926(21,77 \mathrm{~m}), 1912(24,84 \mathrm{~m}), 1992(25,42 \mathrm{~m}), 1964(25,91 \mathrm{~m})$ and $1980(26,00 \mathrm{~m})$. The five larger differences reached in a same year among the maximum and minimum quotas happened in: $1997(14,62 \mathrm{~m}), 1909(14,13 \mathrm{~m}), 1953(12,62 \mathrm{~m}), 1952(12,44 \mathrm{~m})$ and $1916(12,21 \mathrm{~m})$. And the smallest ones happened in: $1912(05,45 \mathrm{~m}), 1968(06,10 \mathrm{~m}), 1985$ $(06,53 \mathrm{~m}), 1974,(06,62 \mathrm{~m})$ and $1986(06,74 \mathrm{~m})$.

Key words: flooding, flow off, Negro River

Insignificante é o declive da bacia do rio Solimões-Amazonas. Numa distância $3.000 \mathrm{~km}$ entre a fronteira com o Peru e o oceano, a declividade é de somente $65 \mathrm{~m}$ (Meggers, 1977), o que vem dificultando, pela falta de uma tecnologia adequada, o achado preciso da altitude do Porto de
Manaus.

Um dos mais antigos e eficientes serviços de medição de níveis de rios da Amazônia foi criado em 1902 pela companhia inglesa Manaos Harbour Limited, então exploradora do Porto de Manaus. Inicialmente, levando-se em conta o nível médio do mar, foi determinada para o Porto uma altitude

\footnotetext{
'Pesquisador Titular, Coordenação de Pesquisas em Geociências, Instituto Nacional de Pesquisas da Amazônia. Caixa Postal, 478.69.082-000 Manaus, Am., Brasil - e-mail:_nunes@inpa.gov.br ${ }^{2}$ Técnica Nivel III, Coordenação de Pesquisas em Geociências, Instituto Nacional de Pesquisas da Amazônia. Caixa Postal 478. 69.082-000 Manaus, Am, Brasil
} 
de uma centena de metros, que perdurou até 1971, quando foi introduzido o fator de correção $60,61 \mathrm{~m}$, empregado até o presente. Em 1997 John Moody e Bob Meade do U.S. Geological Survey de Denver, Colorado, em colaboração com Valderino Pereira da Silva, do Porto de Manaus e Emanuel Lopes, da Companhia de Pesquisas de Recursos Minerais - CPRM, de Manaus, Amazonas, realizaram uma nova aferição com base em duas marcas geodésicas deixadas pelos ingleses. Esse trabalho ficou conhecido através de um memorando o qual não sofreu publicação formal. Os dois pequenos marcos, em latão, estão localizados, um defronte ao edifício do antigo escritório da Manaos Harbour Limited e o outro, detrás da Igreja Matriz, próximo à confluência da avenida Sete de Setembro com a rua Lobo d'Almada. Disso resultou uma correção de $-4,42 \mathrm{~m}$ na altitude do Porto em relação com a atual. Contudo, advertem que as medições que vem sendo realizadas não devam ser alteradas, pois seriam irrelevantes para a grande maioria daqueles que se utilizam dessas cotações diárias.

As cotas aqui referidas foram obtidas de uma régua calibrada de centímetro em centímetro, localizada no Porto de Manaus, conferidas todas as manhãs.

As cotas trabalhadas foram de 1903 até 1999.

Nas cotas diárias coletadas foram levantadas as cinco maiores enchentes e as cinco maiores vazantes. Em seguida foram as cinco menores enchentes $\mathrm{e}$ as cinco menores vazantes. E, finalmente, as cinco maiores e as cinco menores diferenças entre as enchentes e as vazantes ocorridas em um mesmo ano.

As cinco maiores enchentes e vazantes, as cinco menores enchentes e vazantes e as cinco maiores e menores diferenças entre as enchentes e vazantes ocorridas em um mesmo ano, no periodo de 1903 até 1999 no Porto de Manaus, Amazonas, Brasil.

As cinco maiores cotas de enchentes aconteceram em: 1953 $(29,69 \mathrm{~m}), 1976(29,61 \mathrm{~m}), \quad 1989$

As cinco maiores enchentes e vazantes, as cinco menores enchentes e vazantes e as cinco maiores e menores diferenças entre as enchentes e vazantes ocorridas em um mesmo ano, no periodo de 1903 até 1999 no Porto de Manaus, Amazonas, Brasil.

\begin{tabular}{|c|c|c|c|c|c|c|}
\hline ANO & MAX. & DATA & MIN. & DATA & DIFERENÇA & OBSERVAÇÕES \\
\hline 1903 & $27,52 \mathrm{~m}$ & 25-Jun & $16,25 \mathrm{~m}$ & 09-Nov & $11,27 m$ & \\
\hline 1904 & $28,78 \mathrm{~m}$ & 27-Jun & $17,69 \mathrm{~m}$ & 05-07/dez & $11,09 \mathrm{~m}$ & \\
\hline 1905 & $26.07 \mathrm{~m}$ & 22-Jun & $17,52 \mathrm{~m}$ & $10-14 / 0 u t$ & $08,55 \mathrm{~m}$ & \\
\hline 1906 & $26,01 \mathrm{~m}$ & 05-6/Jun & $14.20 \mathrm{~m}$ & 13-Nov & $11,81 \mathrm{~m}$ & 3a.> V \\
\hline 1907 & $27,19 \mathrm{~m}$ & 09-13-Jun & $16,44 m$ & $09-10$ Nov & $10,75 \mathrm{~m}$ & \\
\hline 1908 & $28,92 \mathrm{~m}$ & 09-11/Jun & $18,09 m$ & 30-Out & $10,83 \mathrm{~m}$ & \\
\hline 1909 & $29,17 \mathrm{~m}$ & 14-Jun & $15,04 \mathrm{~m}$ & 23-Out & $\underline{14.13 \mathrm{~m}}$ & $5 \mathrm{a} .>\mathrm{E} \quad 3 \mathrm{a} .>\mathrm{D}$ \\
\hline
\end{tabular}


Continuação

\begin{tabular}{|c|c|c|c|c|c|c|}
\hline 1910 & $27,81 \mathrm{~m}$ & $02-05 / \mathrm{Jul}$ & $18,39 \mathrm{~m}$ & 01-Nov & $09,42 \mathrm{~m}$ & \\
\hline 1911 & $27,57 m$ & $22-J$ un & $16,08 \mathrm{~m}$ & 23-24/Out & $11,49 m$ & \\
\hline 1912 & $24.84 \mathrm{~m}$ & $19-22 / J$ un & $19,43 m$ & 30-Nov & $\underline{05.45 \mathrm{~m}}$ & $2 a .<E \quad 1 a .<D$ \\
\hline 1913 & $28,50 \mathrm{~m}$ & $29-30 / \mathrm{J}$ un & $21.24 \mathrm{~m}$ & 14-Nov & $07,26 \mathrm{~m}$ & $3 a .<V$ \\
\hline 1914 & $28,44 m$ & $17-23 / J$ un & $17,49 \mathrm{~m}$ & $12-14 / \mathrm{Dez}$ & $10,94 m$ & \\
\hline 1915 & $27.73 \mathrm{~m}$ & 27-31/Mai & $16,62 m$ & $06-08 \mathrm{~N}$ ov & $11,11 \mathrm{~m}$ & \\
\hline 1916 & $26,63 \mathrm{~m}$ & $08-10 / \mathrm{Jun}$ & $14.42 \mathrm{~m}$ & 17-Out & $12,21 \mathrm{~m}$ & $4 a .>V$ \\
\hline 1917 & $26,77 \mathrm{~m}$ & 13-15/J un & $17,48 \mathrm{~m}$ & 14-15/Out & $09,29 \mathrm{~m}$ & \\
\hline 1918 & $28,74 m$ & $13-17 / \mathrm{J}$ un & $18,49 m$ & 15-Out & $10,26 \mathrm{~m}$ & \\
\hline 1919 & $26,36 \mathrm{~m}$ & $09-\mathrm{J}$ un & $16,76 \mathrm{~m}$ & 26-Out & $09,60 \mathrm{~m}$ & \\
\hline 1920 & $28,57 m$ & 06-Jul & $19,80 \mathrm{~m}$ & 15-Dez & $08,77 \mathrm{~m}$ & \\
\hline 1921 & $28,97 \mathrm{~m}$ & 13-17/Jun & $17,32 \mathrm{~m}$ & $29-31 / 10-1 / 11$ & $11,65 \mathrm{~m}$ & \\
\hline 1922 & $\underline{29,35 \mathrm{~m}}$ & $18-19 / J$ un & $20,49 m$ & 22-Nov & $08,45 \mathrm{~m}$ & $4 a .>E$ \\
\hline 1923 & $28,19 m$ & $24-J$ un & $16,75 \mathrm{~m}$ & 30-Nov & $11,44 m$ & \\
\hline 1924 & $26,09 \mathrm{~m}$ & 05-06/Jul & $17,31 \mathrm{~m}$ & 01-02/Out & $08,78 \mathrm{~m}$ & \\
\hline 1925 & $28,43 m$ & $29-30 / 6-1-3 / 7$ & $17,67 \mathrm{~m}$ & $16-17 \mathrm{Nov}$ & $10,76 \mathrm{~m}$ & \\
\hline 1926 & $\underline{21} .77 \mathrm{~m}$ & $05-07 / \mathrm{Jul}$ & $14.54 \mathrm{~m}$ & $12-13 /$ Out & $07,23 \mathrm{~m}$ & 1a. $<\mathrm{E} \quad 5 \mathrm{a} .>\mathrm{V}$ \\
\hline 1927 & $27,56 \mathrm{~m}$ & $15-J$ un & $18,78 \mathrm{~m}$ & $22-23 /$ Out & $08,78 \mathrm{~m}$ & \\
\hline 1928 & $28,50 \mathrm{~m}$ & $15-J$ un & $18,19 \mathrm{~m}$ & 05-06/Out & $10,31 \mathrm{~m}$ & \\
\hline 1929 & $28,14 m$ & $20-21 / J$ un & $16.98 \mathrm{~m}$ & 04-Nov & $11,16 \mathrm{~m}$ & \\
\hline 1930 & $27,69 \mathrm{~m}$ & 23-Jun & $18,36 \mathrm{~m}$ & $27-29$ Nov & $09,33 \mathrm{~m}$ & \\
\hline 1931 & $26,66 \mathrm{~m}$ & 06-09/J un & $17,48 \mathrm{~m}$ & 03-Out & $09,18 \mathrm{~m}$ & \\
\hline 1932 & $27,76 \mathrm{~m}$ & $12-16 / J$ un & $17,87 \mathrm{~m}$ & $30-31 / 10 \mathrm{e} 01 / 11$ & $09,89 \mathrm{~m}$ & \\
\hline 1933 & $28,12 \mathrm{~m}$ & 23-24/J un & $16,43 m$ & $25-26 / O$ ut & $11,69 m$ & \\
\hline 1934 & $27,64 m$ & $26-29 / J$ un & $21,16 \mathrm{~m}$ & $25-26 /$ ut & $06,48 m$ & $4 a .<$ vazante $=1973$ \\
\hline 1935 & $27,67 \mathrm{~m}$ & 15-17/J un & $16,15 \mathrm{~m}$ & $05-07 \mathrm{Nov}$ & $11,52 \mathrm{~m}$ & \\
\hline 1936 & $26,64 \mathrm{~m}$ & $20-25 / \mathrm{Mai}$ & $14,97 \mathrm{~m}$ & 29-Set & $11,67 \mathrm{~m}$ & \\
\hline 1937 & $26,91 \mathrm{~m}$ & 19-21/Jun & $16,12 \mathrm{~m}$ & 13-Dez & $10,79 \mathrm{~m}$ & \\
\hline 1938 & $27,92 \mathrm{~m}$ & 15-19/J un & $17,96 \mathrm{~m}$ & 18-19/Out & $09,96 \mathrm{~m}$ & \\
\hline 1939 & $28,03 \mathrm{~m}$ & $17-25 / \mathrm{J}$ un & $20,56 \mathrm{~m}$ & 16-Dez & $07,47 \mathrm{~m}$ & \\
\hline
\end{tabular}


Continuação

\begin{tabular}{|c|c|c|c|c|c|c|c|}
\hline 1940 & $26,77 \mathrm{~m}$ & $30 / 6 e 1-2 / J$ un & $19,58 m$ & 14-Dez & $07,19 \mathrm{~m}$ & & \\
\hline 1941 & $27,09 \mathrm{~m}$ & $28-31 / 5 e 1-2 / J$ un & $16,20 \mathrm{~m}$ & 22-Out & $10,89 \mathrm{~m}$ & & \\
\hline 1942 & $27,63 \mathrm{~m}$ & $25-26 / J$ un & $17,34 \mathrm{~m}$ & 23-Out & $10,29 m$ & & \\
\hline 1943 & $28,19 \mathrm{~m}$ & $30 / 05 \mathrm{e} 1 / \mathrm{Jun}$ & $16,84 m$ & 06-Nov & $11,35 \mathrm{~m}$ & & \\
\hline 1944 & $28,79 m$ & $22-24 / J$ un & $18,11 \mathrm{~m}$ & $17-20 /$ Nov & $10,68 \mathrm{~m}$ & & \\
\hline 1945 & $27,03 \mathrm{~m}$ & $18-21 / J$ un & $16,72 \mathrm{~m}$ & 20-Out & $10,31 \mathrm{~m}$ & & \\
\hline 1946 & $27,98 \mathrm{~m}$ & 08-09/Jun & $17,62 \mathrm{~m}$ & $05-07 / \mathrm{Nov}$ & $10,36 \mathrm{~m}$ & & \\
\hline 1947 & $26,75 \mathrm{~m}$ & $09-11 / J u l$ & $19,49 m$ & $24-25 /$ Out & $07,26 \mathrm{~m}$ & & \\
\hline 1948 & $27,51 \mathrm{~m}$ & 16-Jun & $15,69 \mathrm{~m}$ & 18-19/0ut & $11,82 \mathrm{~m}$ & & \\
\hline 1949 & $28,32 m$ & 18 -Jun & $20,08 \mathrm{~m}$ & 03-Nov & $08,24 m$ & & \\
\hline 1950 & $28,25 m$ & $17-20 / J$ un & $15,74 \mathrm{~m}$ & $09-11 / \mathrm{Nov}$ & $12,51 \mathrm{~m}$ & & \\
\hline 1951 & $28,47 \mathrm{~m}$ & $03-06 / \mathrm{Jul}$ & $18,05 \mathrm{~m}$ & $07-09 / \mathrm{Nov}$ & $10,42 \mathrm{~m}$ & & \\
\hline 1952 & $27,58 \mathrm{~m}$ & $07-11 / \mathrm{J}$ un & $15,14 \mathrm{~m}$ & 30-Out & $12,44 m$ & 5a.> D & \\
\hline 1953 & $\underline{29.69 \mathrm{~m}}$ & $11-12 / J$ un & $17,07 \mathrm{~m}$ & $31 / 10 \mathrm{e} 01 / 11$ & $12.62 \mathrm{~m}$ & 1a.>E & 4a.> D \\
\hline 1954 & $28,49 m$ & $14-23 / J$ un & $17,63 \mathrm{~m}$ & 18-Out & $10,86 \mathrm{~m}$ & & \\
\hline 1955 & $28,53 \mathrm{~m}$ & $21-28 / J$ un & $16,03 \mathrm{~m}$ & 24-Nov & $12,50 \mathrm{~m}$ & & \\
\hline 1956 & $27,65 \mathrm{~m}$ & $23-26 / J$ un & $20,89 \mathrm{~m}$ & 22-Out & $06,76 \mathrm{~m}$ & & \\
\hline 1957 & $27,33 m$ & 09-Jul & $16,51 \mathrm{~m}$ & $21-22 / O u t$ & $10,82 \mathrm{~m}$ & & \\
\hline 1958 & $27,58 \mathrm{~m}$ & $29-31 / 5 e 1-4 / 6$ & $14,74 \mathrm{~m}$ & $18-20 / 0$ ut & $12,84 m$ & & \\
\hline 1959 & $27,71 \mathrm{~m}$ & $30-31 / 6 \mathrm{e} 1 / 07$ & $18,67 \mathrm{~m}$ & 29-31/Out & $09.04 \mathrm{~m}$ & & \\
\hline 1960 & $27,55 \mathrm{~m}$ & $21-23 / J$ un & $18,33 \mathrm{~m}$ & 01-04/Nov & $09,22 \mathrm{~m}$ & & \\
\hline 1961 & $27,13 \mathrm{~m}$ & 07-Jul & $15,96 \mathrm{~m}$ & 12-14/Out & $11,17 \mathrm{~m}$ & & \\
\hline 1962 & $28,33 m$ & 04-06/Jul & $17.15 \mathrm{~m}$ & 25-26/Out & $11.18 \mathrm{~m}$ & & \\
\hline 1963 & $27,31 \mathrm{~m}$ & $17-19 / \mathrm{J}$ un & $13,65 \mathrm{~m}$ & $30-31 / 0$ ut & $13,66 \mathrm{~m}$ & $1 a,>V$ & \\
\hline 1964 & $25.91 \mathrm{~m}$ & $13-14 / \mathrm{Jul}$ & $18,41 \mathrm{~m}$ & 28-Nov & $07,50 \mathrm{~m}$ & $4 a .<E$ & \\
\hline 1965 & $26,58 \mathrm{~m}$ & $14-18 / \mathrm{J}$ un & $16,60 \mathrm{~m}$ & O6-Nov & $09,98 \mathrm{~m}$ & & \\
\hline 1966 & $26,41 \mathrm{~m}$ & $20-22 / J$ un & $16,76 \mathrm{~m}$ & 11-Nov & $09,65 \mathrm{~m}$ & & \\
\hline 1967 & $27,91 \mathrm{~m}$ & $19-20 / \mathrm{J}$ un & $16,18 \mathrm{~m}$ & 25-27/Out & $11.76 \mathrm{~m}$ & & \\
\hline 1968 & $27,13 \mathrm{~m}$ & 04-J un & $21,03 m$ & 31-Dez & $06.10 \mathrm{~m}$ & 2a. $<$ D & \\
\hline 1969 & $27,40 \mathrm{~m}$ & $26-31 / \mathrm{Mai}$ & $16,86 \mathrm{~m}$ & 02-05/Dez & $10,54 \mathrm{~m}$ & & \\
\hline
\end{tabular}


Continuação

\begin{tabular}{|c|c|c|c|c|c|c|c|}
\hline 1970 & $28,31 \mathrm{~m}$ & 26-28/Jun & $18,19 \mathrm{~m}$ & $12-13$ N ov & $10,02 m$ & & \\
\hline 1971 & $29,12 m$ & 24-28/Jun & $21,14 \mathrm{~m}$ & 08-Nov & $07,98 \mathrm{~m}$ & & \\
\hline 1972 & $28,70 \mathrm{~m}$ & $16-22 / J$ un & $20,02 \mathrm{~m}$ & $10-12$ Nov & $08,68 \mathrm{~m}$ & & \\
\hline 1973 & $28,57 \mathrm{~m}$ & $06-07 / \mathrm{Jul}$ & $\underline{21,16 \mathrm{~m}}$ & $20-21$ Nov & $07,41 \mathrm{~m}$ & $4 \mathrm{a}<\mathrm{V}=$ & 1934 \\
\hline 1974 & $28,46 \mathrm{~m}$ & $02-04 / J u l$ & $\underline{21,84 \mathrm{~m}}$ & $05-10 / \mathrm{Dez}$ & $\underline{06.62 \mathrm{~m}}$ & $1 a<<$ & $4 \mathrm{a} .<\mathrm{D}$ \\
\hline 1975 & $29,11 \mathrm{~m}$ & 23-25/J un & $19,32 m$ & $28-29$ Nov & $09,79 m$ & & \\
\hline 1976 & $\underline{29,61 \mathrm{~m}}$ & 14-17/Jun & $18,05 \mathrm{~m}$ & 22-Nov & $11,56 \mathrm{~m}$ & $2 a .>E$ & \\
\hline 1977 & $28,45 \mathrm{~m}$ & $28-29 / J$ un & $20,66 \mathrm{~m}$ & 14-15/Out & $07,79 \mathrm{~m}$ & & \\
\hline 1978 & $28,11 \mathrm{~m}$ & 18-20/Jun & $20,12 \mathrm{~m}$ & $05-07 / \mathrm{Dez}$ & $07,99 \mathrm{~m}$ & & \\
\hline 1979 & $28,23 m$ & $25-26 / J$ un & $17,44 \mathrm{~m}$ & 22-Out & $10,79 m$ & & \\
\hline 1980 & $\underline{26.00 \mathrm{~m}}$ & 01-03/Jul & $17,68 \mathrm{~m}$ & $08-12 /$ Out & $08,32 \mathrm{~m}$ & 5a. $<\mathrm{E}$ & \\
\hline 1981 & $26,85 \mathrm{~m}$ & 22-23/Jun & $17,24 \mathrm{~m}$ & $12-15 \mathrm{Nov}$ & $09,61 \mathrm{~m}$ & & \\
\hline 1982 & $28,97 \mathrm{~m}$ & $22-26 / \mathrm{J}$ un & $18,28 \mathrm{~m}$ & $02-04$ Nov & $10,69 \mathrm{~m}$ & & \\
\hline 1983 & $26,52 \mathrm{~m}$ & 06-08/Jun & $17,08 \mathrm{~m}$ & $24-25 / O u t$ & $09,44 m$ & & \\
\hline 1984 & $28,03 \mathrm{~m}$ & 18-23/Jun & $19,58 \mathrm{~m}$ & $31 / 10 \mathrm{e} 01 / 11$ & $08,45 \mathrm{~m}$ & & \\
\hline 1985 & $26,27 m$ & $01-04 / \mathrm{Jul}$ & $19,74 \mathrm{~m}$ & $27-28 \mathrm{Nov}$ & $06.53 \mathrm{~m}$ & $3 a_{0}<D$ & \\
\hline 1986 & $28,14 \mathrm{~m}$ & 16-18/Jul & $21.40 \mathrm{~m}$ & 13-15/Out & $06.74 \mathrm{~m}$ & $2 a_{.}<\mathrm{V}$ & $5 a_{0}<D$ \\
\hline 1987 & $27,91 \mathrm{~m}$ & $09-11 / \mathrm{J}$ un & $17,99 m$ & $06-10$ Nov & $09,92 \mathrm{~m}$ & & \\
\hline 1988 & $27,78 \mathrm{~m}$ & $20-30 / J$ un & $17,82 \mathrm{~m}$ & $12-14 /$ Out & $09,96 \mathrm{~m}$ & & \\
\hline 1989 & $\underline{29,42 \mathrm{~m}}$ & 03-06/Jul & $19,55 \mathrm{~m}$ & 31-Dez & $09,87 \mathrm{~m}$ & $3 a .>E$ & \\
\hline 1990 & $28,23 \mathrm{~m}$ & $17-19 / \mathrm{J}$ un & $16,32 \mathrm{~m}$ & $01-02 / N o v$ & $11,91 \mathrm{~m}$ & & \\
\hline 1991 & $28,06 \mathrm{~m}$ & $05-07 / \mathrm{Jul}$ & $16,07 \mathrm{~m}$ & $05-07 \mathrm{Nov}$ & $11,99 \mathrm{~m}$ & & \\
\hline 1992 & $25,42 \mathrm{~m}$ & $20-24 / \mathrm{Mai}$ & $17,56 \mathrm{~m}$ & $12-13$ Nov & $07,86 \mathrm{~m}$ & $3 a_{0}<\mathrm{E}$ & \\
\hline 1993 & $28,76 \mathrm{~m}$ & $09-10 / \mathrm{J}$ un & $19,46 \mathrm{~m}$ & 27-28/Out & $09,29 \mathrm{~m}$ & & \\
\hline 1994 & $29,05 \mathrm{~m}$ & 26-27/Jun & $19,06 \mathrm{~m}$ & $25-30$ Nov & $09,99 \mathrm{~m}$ & & \\
\hline 1995 & $27,16 \mathrm{~m}$ & 29-30/J un & $15,06 \mathrm{~m}$ & 30-Out & $12,10 \mathrm{~m}$ & & \\
\hline 1996 & $28,54 \mathrm{~m}$ & $09-12 / \mathrm{J}$ un & $19,14 m$ & $19-23 /$ Out & $09,40 \mathrm{~m}$ & & \\
\hline 1997 & $28,96 m$ & $10-12 / \mathrm{J}$ un & $\underline{14,34 \mathrm{~m}}$ & 04-05Nov & $\underline{14.62 \mathrm{~m}}$ & $2 a .>V$ & 1a. $>D$ \\
\hline 1998 & $27,58 \mathrm{~m}$ & $06-07 / J u l$ & $20,16 \mathrm{~m}$ & $30-31 /$ ut & $07,42 m$ & & \\
\hline 1999 & $29,30 \mathrm{~m}$ & 24-28/J un & 16,95 & 22-23/Out & $12,95 \mathrm{~m}$ & 5a. $>\mathrm{E}$ & $2 a .>D$ \\
\hline
\end{tabular}

Os dados acima foram fornecidos pelo Serviço de Engenharia da Sociedade de Navegaçáo, Porto e Hidrovia do Amazonas

$E=$ Enchente $\quad \mathrm{V}=$ Vazante $\quad \mathrm{D}=$ Diferença $\quad>=$ Maior $\quad<=$ Menor 
$(29,42 \mathrm{~m}), 1922(29,35 \mathrm{~m})$ e 1909 $(29,17 \mathrm{~m})$.

As cinco maiores cotas de vazantes aconteceram em: 1963 (13,65m), $1906(14,20 \mathrm{~m}), 1997$ $(14,34 \mathrm{~m}), 1916(14,42 \mathrm{~m})$ e 1926 (14,54m).

Os cinco menores valores de cotas máximas foram: $1926(21,77 \mathrm{~m})$, $1912(24,84 m), 1992(25,42 m), 1964$ $(25,91 \mathrm{~m})$ e $1980(26,00 \mathrm{~m})$.

As cinco maiores diferenças alcançadas entre as cotas de cheias e as cotas de vazante foram: 1997 $(14,62 \mathrm{~m}), 1909(14,13 \mathrm{~m}), 1953$ $(12,62 \mathrm{~m}), 1952(12,44 \mathrm{~m})$ e 1916 $(12,21 \mathrm{~m})$ e as cinco menores:1912 $(05,45 \mathrm{~m}), 1968(06,10 \mathrm{~m}), 1985$ $(06,53 \mathrm{~m}), 1974(06,62 \mathrm{~m})$ e 1986 $(06,74 \mathrm{~m})$.

Um fato curioso aconteceu nas vazantes de 1934 e 1973, 39 anos de diferença, ambas atingindo a cota de $21,16 \mathrm{~m}$, a $4^{\mathrm{a}}$ menor vazante registrada.

No que se refere às enchentes ocorridas no final de 1800 e início do século passado temos o relato de Campos (1910), embora não existisse ainda uma régua aferida, ele cita como enormes às de 1883, 1890, $1897 \mathrm{e}$ 1904, ocorridas numa seqüência de sete em sete anos. Refere-se ainda, que era esperada outra grande enchente para 1911, a qual veio a acontecer em 1908, antecipando-se em três anos. Como a régua do Porto somente foi instalada em 1902, nada teriamos para avaliar os dados de Campos (op. cit.), caso não houvesse feito referências sobre as enchentes de 1904 $(28,78 \mathrm{~m})$ e a de $1908(28,92 \mathrm{~m})$ naturalmente com informações retiradas dos arquivos do Porto. No quadro, nenhuma das duas está incluída entre as cinco maiores cotas, o que nos leva a inferir como cheias excepcionais, toda aquelas iguais ou superiores à cota de $29,00 \mathrm{~m}$. A edição dessa obra consultada foi a segunda, datada de 1910, dai, talvez a ausência de referência sobre a grande enchente de 1909 , que atingiu a cota de $29,17 \mathrm{~m}$. Um outro fato interessante é que naquela obra não são encontradas informações sobre as secas.

Está na lembrança das pessoas mais antigas, a grande enchente de 1922, que atingiu a cota máxima de $29,35 \mathrm{~m}$, hegemonia que duraria trinta anos, até ser suplantada pela de 1953 $(29,69 \mathrm{~m})$, a maior do século. Com a enchente de $1976(29,61 \mathrm{~m})$ vinte e cinco anos depois, passaria para o terceiro lugar e mais treze anos, com a de $1989(29,42 \mathrm{~m})$, para chegar ao atual quarto lugar.

Qualquer tentativa de prever-se futuras enchentes ou vazantes com base em análises estatísticas ou probabilísticas parece laborar em erro, uma vez que as previsões metereológicas não se enquadram em pensamentos matemáticos. $\mathrm{O}$ fenômeno El Niño acontecido em 1997, parece haver produzido uma seca ou vazante acentuada. Mais uma vez, esse fenômeno mundial, que se repete em periodo variável de 7 a 14 anos, vem tornando muito difícil prever-se as ocorrências do futuro. A El Niña ocasionaria fenômeno oposto, cheias portanto, poderia haver provocado as enchentes referidas por 
Campos (1910) ocorridas no século passado, para não mais se repetir.

\section{Bibliografia citada}

Campos, H.L. 1910. Climatologia Médica do Estado do Amazonas. $2^{x}$ edição. Secção de Obras da Imprensa Official, Manáos, Amazonas.

Meggers, B.J. 1977. A Amazônia a ilusào de um paraiso. Editora Civilização Brasileira S.A. 207 p.. Tradução de Maria Yedda Linhares.

Moody, J.; Meade, B. 1997. Gage Datam at Manaus. Mem. de 09 de março de 1997. U.S.Geological Survey, 80225 Denver, Colorado. 